\title{
Transition to chaos and escape phenomenon in two-degrees-of-freedom oscillator with a kinematic excitation
}

\author{
Marek Borowiec • Grzegorz Litak
}

Received: 11 March 2012 / Accepted: 19 June 2012 / Published online: 12 July 2012

(C) The Author(s) 2012. This article is published with open access at Springerlink.com

\begin{abstract}
We study the dynamics of a two-degreesof-freedom (two-DOF) nonlinear oscillator representing a quarter-car model excited by a road roughness profile. Modeling the road profile by means of a harmonic function, we derive the Melnikov criterion for a system transition to chaos or escape. The analytically obtained estimations are confirmed by numerical simulations. To analyze the transient vibrations, we used recurrences.
\end{abstract}

Keywords Nonlinear oscillations · Vehicle suspension - Recurrence plot

\section{Introduction}

The dynamics of a quarter-car model is governed by the road profile excitations and nonlinear suspension characteristics $[1-3,5]$. These elements were discussed separately using simplified models and jointly by considering the more realistic description of a vehicle motion. For instance, Verros et al. [1] proposed a quarter-car model with piecewise linear dynamical characteristics. According to the adapted control

M. Borowiec · G. Litak ( $\varangle)$

Department of Applied Mechanics, Lublin University of Technology, Nadbystrzycka 36, 20-618 Lublin, Poland e-mail: g.litak@pollub.pl strategy, the damping coefficient switched between two different values. Gobbi and Mastinu [2] considered a two-DOF model to derive a number of analytical formulae describing the dynamic behavior of passively suspended vehicles running on randomly profiled roads. Their linear model approach was generalized by Von Wagner [3], who determined a corresponding high-dimensional probability density by solving the Fokker-Planck equations. Finally, in papers [4-6] dynamics, bifurcations, and appearance of chaotic solutions were discussed.

However, the main issues of vehicle dynamics studies were unwanted and harmful vibration responses generated by vehicle as an effect of a rough surface road profile kinematic excitation [1, 4, 7-11]. Thus, the efficient reduction of them is still a subject of research among automotive manufacturers and research groups [14, 15]. Turkay and Akcay [7] considered constraints on the transfer functions from the road disturbance to the vertical acceleration, the suspension travel, and the tire deflection are derived for a quarter-car active suspension system using the vertical acceleration and/or the suspension travel measurements for feedback. The recent experimental and theoretical studies involved many new applications of active and semi-active control procedures [12-16]. Consequently, previous mechanical quarter-car models $[3,10,11,15]$ have been reexamined in the context of active damper applications. Dampers based on a magnetorheological fluid with typical hysteretic char- 


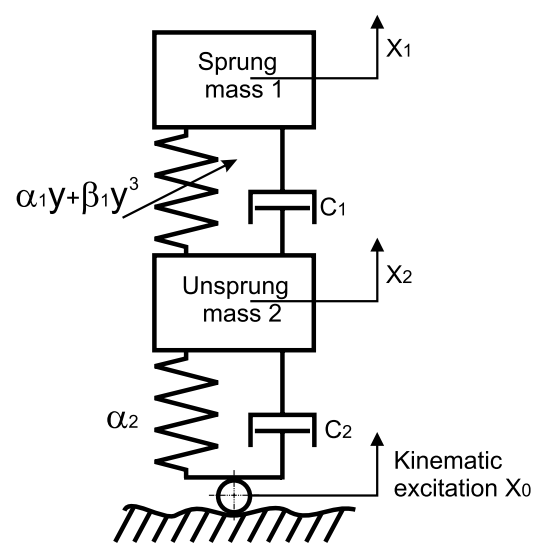

Fig. 1 Two-degree-of-freedom quarter-car model $\left(y=x_{1}-x_{2}\right)$

acteristics have a significant expectation for effective vibration damping in many applications [4, 17-23].

Recent efforts have been also focused on studies of the excitation of an automobile by a road surface profile with harmful noise components [3, 24, 25]. Noise-like chaotic vibrations, appearing due to the system nonlinearities, have been investigated in simplified single-DOF models $[4,6,19]$. These papers follow the rich literature on an escape phenomenon in the symmetric and nonsymmetric Duffing or Helmholtz potentials [26-30], where critical system parameters were determined. Note that the condition for escape could be applied as a criterion of fractality in basins of attraction and also as a transition to chaos [29]. On the other hand, more sophisticated models of vehicle dynamics, namely half-car and full-car models, in the context of nonlinear response including chaotic solutions have been studied by Zhu and Ishitobi and also by Wang et al. [31-33].

To study a transition to the chaotic region and the corresponding critical parameters in a low-dimensional dynamical system analytically, the Melnikov theory [34-38] is often advocated. The application of this approach to a simple quarter-car model has been recently proposed by Li et al. [4] and Litak et al. [19]. In the above papers, a single-DOF model was used because of its simplicity. Consequently, the analytic consideration included also multiple-scale analysis and harmonic balance [20,39]. The present paper is a continuation of the previous studies with an extension to a more realistic two-DOF model, which includes the sprung and unsprung masses (Fig. 1).

Our article is organized in four sections. After introduction in the present section (Sect. 1) we present the model and discuss possibility of a global homoclinic bifurcation (Sect. 2). By the reduction of dimension we introduce the foundations of the Melnikov approach to the model. In this section (Sect. 2) we obtain the principal result as a critical curve which defines the system parameter regions of regular and nonperiodic (chaos, transient chaos, or escape) behavior. The simulation results illustrating those transition results are also shown. Further results including recurrence analysis confirming the theoretical predictions are provided in Sect. 3. Finally, in Sect. 4 we end up with conclusions and final remarks.

\section{The model and global bifurcations}

We start the analysis from the two-DOF model presented in Fig. 1. The dynamics of vehicle excited by the road profile quarter-car model is governed by nonlinear suspension characteristics. To examine a transition to the chaotic regime of vibrations, the Melnikov theory has been recently proposed [4, 19]. In this perturbation approach the authors of previous works used a single-DOF model.

In the present note we go beyond this assumption by considering an extension of a vehicle model with the defined unsprung and sprung masses (Fig. 1). The differential equations of motion for both masses have the following form:

$$
\begin{aligned}
& \frac{\mathrm{d}^{2}}{\mathrm{~d} t^{2}} x_{1}+\frac{c_{1}}{m_{1}}\left(\frac{\mathrm{d}}{\mathrm{d} t} x_{1}-\frac{\mathrm{d}}{\mathrm{d} t} x_{2}\right)+\frac{\alpha_{1}}{m_{1}}\left(x_{1}-x_{2}\right) \\
& \quad+\frac{\beta_{1}}{m_{1}}\left(x_{1}-x_{2}\right)^{3}=0, \\
& \frac{\mathrm{d}^{2}}{\mathrm{~d} t^{2}} x_{2}+\frac{c_{2}}{m_{2}}\left(\frac{\mathrm{d}}{\mathrm{d} t} x_{2}-\frac{\mathrm{d}}{\mathrm{d} t} x_{0}\right)-\frac{c_{1}}{m_{2}}\left(\frac{\mathrm{d}}{\mathrm{d} t} x_{1}-\frac{\mathrm{d}}{\mathrm{d} t} x_{2}\right) \\
& \quad+\frac{\alpha_{2}}{m_{2}}\left(x_{2}-x_{0}\right)-\frac{\alpha_{1}}{m_{2}}\left(x_{1}-x_{2}\right) \\
& -\frac{\beta_{1}}{m_{2}}\left(x_{1}-x_{2}\right)^{3}=0,
\end{aligned}
$$

where $m_{1}$ and $m_{2}$ denote the corresponding sprung and unsprung masses (Fig. 1), and $x_{0}=a \cos \omega t$ describes the harmonic corrugation of a road profile. $c_{i}$, $\alpha_{i}$ (for $i=1,2$ ) and $\beta_{1}$ are damping and stiffness coefficients, respectively.

Now we define the new variable $y=x_{1}-x_{2}$ of the relative motion:

$\ddot{y}+\epsilon \frac{c_{1}}{m_{1}} \dot{y}+\frac{\alpha_{1}}{m_{1}} y+\frac{\beta_{1}}{m_{1}} y^{3}=\ddot{x}_{2}$. 
Fig. 2 Potential of the restore force- $V(y)$ (Eq. (12)) for different $A_{3}$ (a) and the corresponding heteroclinic orbits (b)

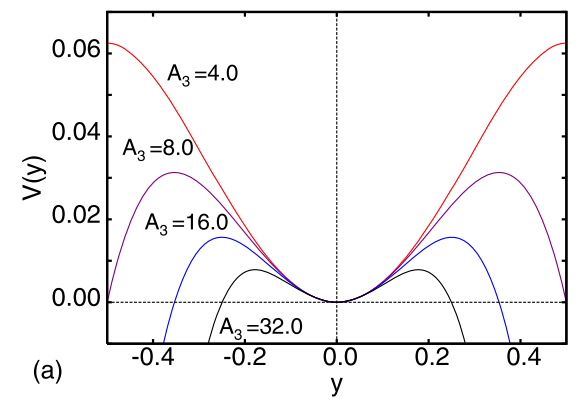

To adjust the above equations to application of the higher-dimensional Melnikov approach [40], the above equations can be approximated by introducing a small parameter $\epsilon$ :

$\ddot{y}+\epsilon \frac{c_{1}}{m_{1}} \dot{y}+\frac{\alpha_{1}}{m_{1}} y+\frac{\beta_{1}}{m_{1}} y^{3}=-\ddot{x}_{2}$.

Consequently, the second equation (Eq. (2)) gets the new form

$$
\begin{aligned}
\ddot{x}_{2} & +\frac{c_{2}}{m_{2}} \dot{x}_{2}+\frac{\alpha_{2}}{m_{2}} x_{2} \\
& =\frac{\alpha_{1}}{m_{2}} y+\frac{\beta_{1}}{m_{2}} y^{3}-\frac{c_{2}}{m_{2}} \omega a \epsilon \sin \omega t+\frac{\alpha_{2}}{m_{2}} a \epsilon \cos \omega t .
\end{aligned}
$$

Using the Melnikov approach [34] in $y$ coordinate equation (Eq. (3)), we study the Hamiltonian system of the nodal kinetic energy defined at the saddle points. In this way, time variability of $y$ and $\dot{y}$ is negligible. The above equations can be rewritten in the dimensionless form:

$$
\begin{aligned}
\ddot{y}+ & \epsilon C_{1} A_{1} \dot{y}+y+A_{3} y^{3}=-\ddot{x}_{2}, \\
\ddot{x}_{2}+ & C_{2} A_{1} \dot{x}_{2}+A_{2} M x_{2} \\
= & C_{1} M \frac{\sqrt{m_{1} \alpha_{1}}}{m_{1}} \dot{y}+M y+A_{3} M y^{3} \\
& \quad-\epsilon C_{2} M a \omega \sin (\omega t)+\epsilon A_{2} M a \cos (\omega t),
\end{aligned}
$$

where $C_{1}=\frac{c_{1}}{\alpha_{1}}, C_{2}=\frac{c_{2}}{\alpha_{1}}, A_{1}=\frac{\sqrt{\alpha_{1}}}{\sqrt{m_{1}}}, A_{2}=\frac{\alpha_{2}}{\alpha_{1}}, A_{3}=$ $\frac{\beta_{1}}{\alpha_{1}}, M=\frac{m_{1}}{m_{2}}$ are dimensionless parameters. For further consideration, we assumed that the system parameters are: $C_{1}=0.001, C_{2}=0.5, A_{1}=1, A_{2}=1$, $A_{3}=-16, M=5$. The excitation frequency and the corresponding amplitudes used in the analysis have been fixed to $\omega=1.5, a=0.08$, and $a=0.12$. In the numerical simulations we used the sampling time $\delta t=0.00418$.
To analyze a homoclinic bifurcation in the sprung mass vibration, we make further approximation. The role of the small parameter $\epsilon$ is to determine the heteroclinic trajectory and decouple the equations of motion into separate equations for sprung and unsprung masses. Thus, after above normalizations the equations can be expressed as

$$
\begin{aligned}
\dot{v}= & -\epsilon C_{1} v-y-A_{3} y^{3}-\ddot{x}_{2}, \\
\dot{y}= & v, \\
\ddot{x}_{2}= & -C_{2} \dot{x}_{2}-A_{2} M x_{2}+M\left(C_{1} v+y+A_{3} y^{3}\right. \\
& \left.\quad-\epsilon C_{2} \omega a \sin \omega t+\epsilon A_{2} a \cos \omega t\right) .
\end{aligned}
$$

Interestingly, in the limit of small $\epsilon$ limit, $x_{2}$ can be approximated as

$x_{2}=\epsilon A \cos (\omega t+\phi-\psi)+x_{s t}$.

Note that in the above expression, $x_{s t}$, generated by slowly changing terms with $y$ and $\dot{y}$, is playing a role of the static displacement, while $A$ is an amplitude.

Thus, the unperturbed equations (for $\epsilon=0$ ) can be obtained from the gradient of the unperturbed Hamiltonian $H^{0}(y, v)$ :

$\dot{y}=\frac{\partial H^{0}}{\partial v}, \quad \dot{v}_{y}=-\frac{\partial H^{0}}{\partial y}$,

where $H^{0}$ is defined as follows:

$H^{0}=\frac{v^{2}}{2}+V(y)$.

The corresponding effective potential (Fig. 2a) is given by the expression

$V(y)=\frac{y^{2}}{2}+A_{3} \frac{y^{4}}{4}$.

Following the standard Melnikov theory [34-38], we get the heteroclinic orbits connecting the two hyperbolic saddle points (coinciding with the maxima of the potential $V(y)$ (Eq. (12)): $y= \pm \sqrt{A_{3}^{-1}}$. 
They can be expressed analytically as

$$
\begin{aligned}
& y^{*}=-\sqrt{A_{3}^{-1}} \tanh \left[\left(t-t_{0}\right) \frac{\sqrt{2 A_{3}}}{2}\right], \\
& v^{*}=\frac{\mathrm{d} y^{*}(t)}{\mathrm{d} t}=-\frac{\sqrt{2}}{2 \cosh ^{2}\left[\left(t-t_{0}\right) \frac{\sqrt{2 A_{3}}}{2}\right]} .
\end{aligned}
$$

After perturbations of the heteroclinic orbits (Fig. 2b), the stable and unstable manifolds are calculated. Because of perturbations, they are detracted (Fig. 3). As the characteristic distance between them $d \rightarrow 0$, the system possibilities of mixed solutions (regular and escape) appear, which is equivalent to irregular chaotic and chaotic transient solutions. Thus, $d=0$ is the ideal criterion for chaos appearance.

Formally, the distance $d$ between perturbed stable and unstable manifolds are proportional to the Melnikov integral (Fig. 3) $d \sim \mathcal{M}\left(t_{0}\right)$ [34-36], which can be written as

$\mathcal{M}\left(t_{0}\right)=\int_{-\infty}^{+\infty} h\left(y^{*}(t), v^{*}(t)\right) \wedge g\left(y^{*}(t), v^{*}(t)\right) \mathrm{d} t$,

where $\wedge$ denotes a wedge product, the differential form $h$ is the gradient of the unperturbed Hamiltonian,

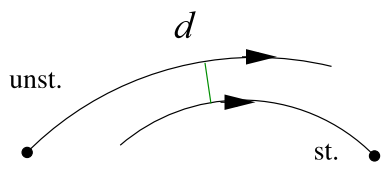

Fig. 3 Stable and unstable manifolds of perturbed orbits terminated and started in the corresponding saddle points, and the distance between them $d$ (in $y-v$ plane)

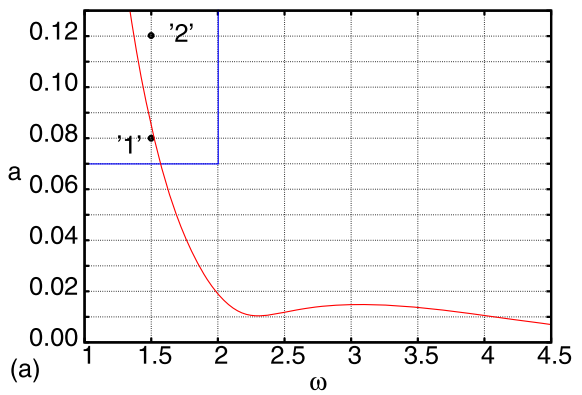

Fig. 4 The Melnikov criterion, $a=\eta C_{1}$ versus $\omega$ for system parameters where $A_{3}=-16, M=5, A_{2}=1.5, C_{1}=0.001$, $C_{2}=0.5$. The curve separate the region of regular solutions (below the curve) from the chaotic and escape solutions (above the curve). Note that (b) magnifies the marked region in (a), $g$ is related to the perturbation part, and $t_{0}$ is an integration constant. Both forms are defined on unperturbed heteroclinic orbit stable and unstable manifolds $W_{\text {st(unst })}=\left(y_{\text {st(unst) }}^{*}, v_{\text {st(unst })}^{*}\right)$ :

$h=v \mathrm{~d} v+\left(-y-A_{3} y^{3}\right) \mathrm{d} y$,

$g=\left(A \omega^{2} \cos (\omega t+\phi-\Psi)-C_{1} v\right) \mathrm{d} y$.

Thus, after substitution the above forms into the Melnikov function $\mathcal{M}\left(t_{0}\right)$ (Eq. (14)), we get:

$$
\begin{aligned}
\mathcal{M}\left(t_{0}\right)= & M \int_{-\infty}^{\infty}\left(-C_{1} v^{*}\right. \\
& \left.+A \omega^{2} \cos (\omega t+\phi-\Psi)\right) v^{*} \mathrm{~d} t .
\end{aligned}
$$

The condition for a global homoclinic transition, corresponding to a possible horse-shoe-type crosssection of stable and unstable manifolds, can be written as

$\bigvee_{t_{0}} \mathcal{M}\left(t_{0}\right)=0 \quad$ and $\quad \frac{\partial \mathcal{M}\left(t_{0}\right)}{\partial t_{0}} \neq 0$.

Consequently, the critical parameter $\eta=a / C_{1}$ is now

$$
\begin{aligned}
\eta= & \frac{2 \sqrt{-A_{3}}}{3 \pi \omega^{3} M} \sqrt{\frac{\left(A_{2} M-\omega^{2}\right)^{2}+C_{2}^{2} \omega^{2}}{A_{2}^{2}+C_{2}^{2} \omega^{2}}} \\
& \times \sinh \left(\frac{\pi \omega}{\sqrt{-2 A_{3}}}\right) .
\end{aligned}
$$

The results of the above analysis are presented in Fig. 4a. The curve separates the region of regular solutions from the chaotic and escape ones. The black

(b)

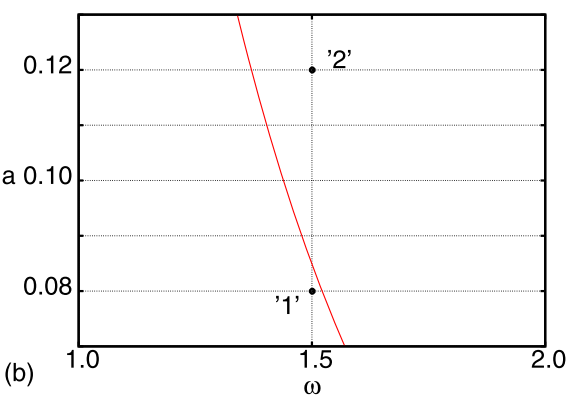

where the two indicated points symbolize the parameters used for numerical calculation points above and below the critical curve for $a=0.08$ at the point " 1 ", and $a=0.12115$ at the point " 2 " ( $\omega=1.5$ for both cases $)$ 


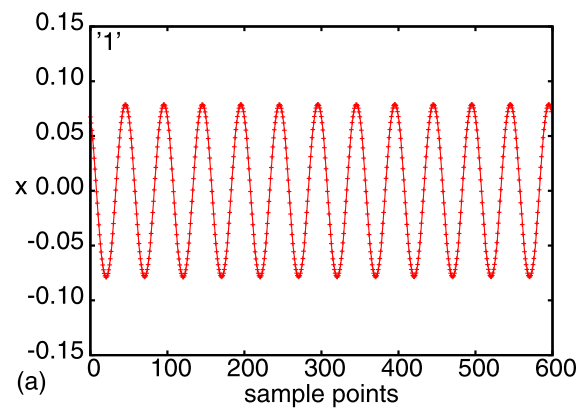

Fig. 5 Time series (displacements of the sprung mass) of regular (a) and irregular (or chaotic transient) (b) solutions for the same system parameters as at points " 1 " and "2" in Figs. 4a

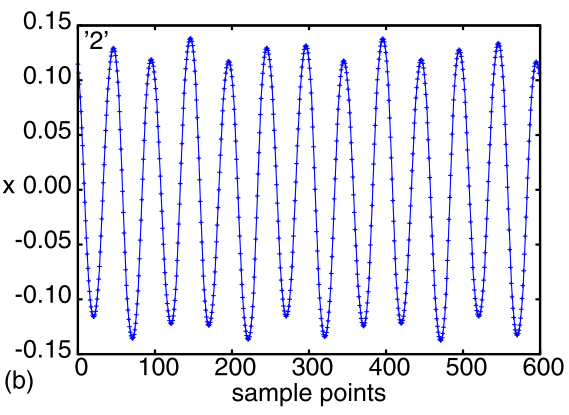

and $4 \mathrm{~b}$, respectively (the sampling time $\delta t=0.00418$ ). Note, the larger amplitude and additional modulation in (b)

Fig. 6 Phase portraits (displacements versus velocity of the sprung mass) of regular (a) and irregular (b) solutions for the same system parameters as point " 1 " and "2" in Figs. 4a and $4 \mathrm{~b}$, respectively
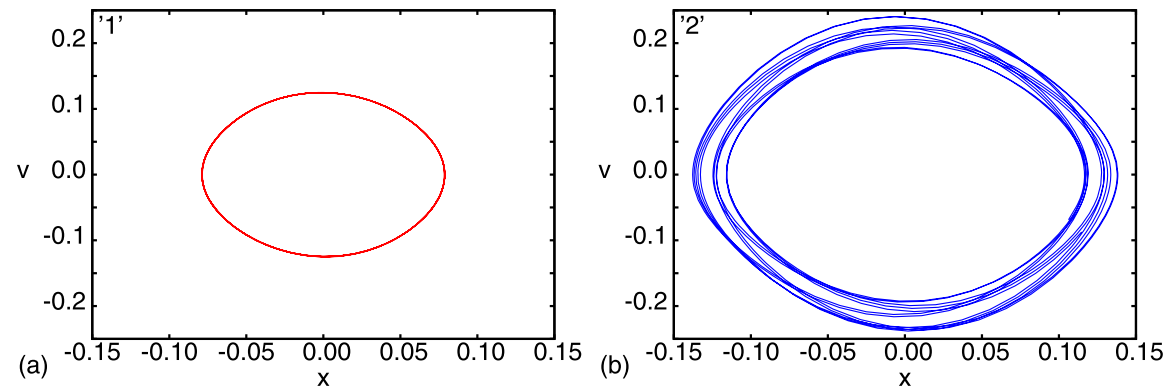

Fig. 7 Time series of displacement (a) and phase portrait (b) during transient vibration escape (corresponding time interval $\Delta t=3844.25$, while sampling time $\delta t=0.00418)$
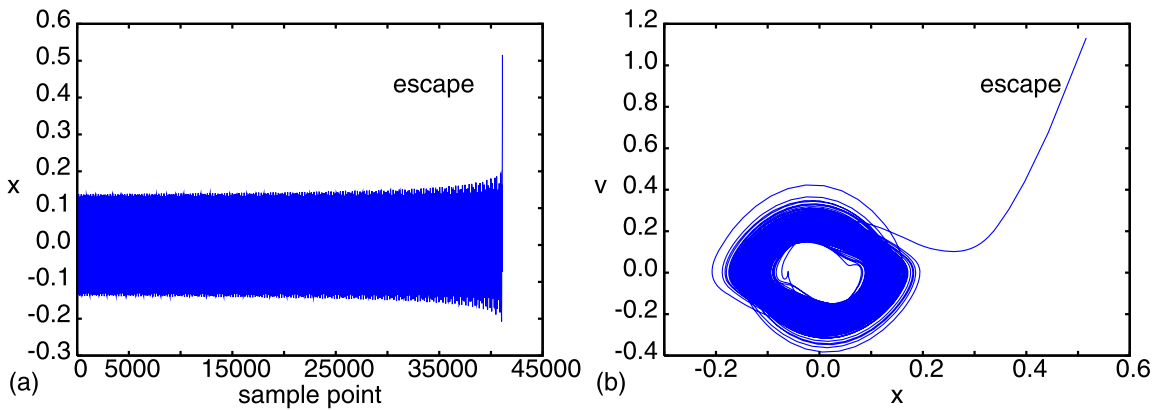

points represent the parameters used for numerical simulations. To show this region of parameters, Fig. $4 \mathrm{~b}$ magnifies the surrounding area. The corresponding time histories are presented in Figs. 5a and 5b. Note that for purpose of simulations, we used Eqs. (7-8) with $\epsilon=1$.

Note that Fig. 5a shows the mono-frequency, while Fig. 5b shows more complex responses (multifrequency or irregular). This is clearly visible in Figs. $6 \mathrm{a}$ and $6 \mathrm{~b}$, where we show the corresponding phase diagrams. In Fig. 6 a one can see a single line, while in Fig. 6b lines are split into characteristic threeline patterns. It is worth mentioning that the Melnikov criterion (Eq. (18) and Fig. 4) specifies the global transition associated with the destruction of borders between basins of attractions belonging to different solutions. In our case, one of basins is related to an escape from the potential well. The irregular solution denoted as no. 2 in Fig. 4 (see also Figs. 5b and 6b) must be related to such an escape. Indeed, continuing the calculations for long enough time interval, one observes the escape in the plot on time series (Fig. 7a) and on the phase portrait (Fig. 7b), respectively.

Focusing on the above solutions, we discuss the recurrence properties of numerical results with more details in the next section. 


\section{Recurrence plot analysis}

The numerical solutions of the regular and transient nature can be analyzed more carefully by the recurrence plots $[41,42]$. This method is based on the statistics of recurrences and can be described by the matrix form $\mathbf{R}^{m, \varepsilon}$ with corresponding 0 and 1 elements:

$R_{i j}^{m, \varepsilon}=\Theta\left(\varepsilon-\left|\mathbf{x}_{i}-\mathbf{x}_{j}\right|\right)$ for $|i-j| \geq w$,

where $\mathbf{x}_{i}$ and $\mathbf{x}_{j}$ are usually defined in the embedding space of dimension $m$, and $\varepsilon$ is the threshold value. Here indices $i$ and $j$ denote the sampling instants. In our case we decided to use the $m=2$ and the embedding space including the displacements of sprung and unsprung masses: $x_{j}=\left[y, x_{2}\right]$ (Eqs. (7-8) with $\epsilon=1$ ).

Having 0 and 1 values, they are translated into the recurrence diagram as an empty place and colored dots, respectively (see Fig. 9). Here $w$ denotes the Theiler window used to exclude identical and neighboring points from the analysis [44]. Webber and Zbilut [45] and later Marwan and collaborators [43, 44] developed the recurrence quantification analysis (RQA) for recurrence plots.

Shortly after its invention, RQA was addressed to the biological and physiologic systems [45, 46]. Recently this method has been applied for several technical systems [47-49]. The first parameter of the RQA

Fig. 8 The idea of states summation idea in the embedded space and the sphere of radius $\varepsilon$

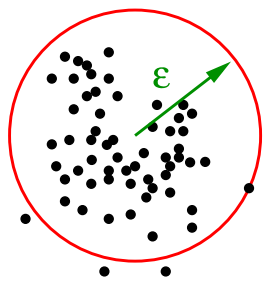

defining the correlation function is the recurrence rate $R R$,

$R R=\frac{1}{N^{2}} \sum_{i, j=1}^{N} R_{i j}^{m, \varepsilon}$,

which calculates the number of recurrences. In our case, the Theiler window $w=0$ in order to get the consistency with correlation sum [44]. In this language, $\varepsilon$ expresses the correlation length of the characteristic sphere radius in the embedded space (Fig. 8). Note that the correlation sum is an important tool which could be used to derive correlation dimension $D_{2}$. For small enough $\varepsilon$,

$D_{2}=\frac{\log R R(\varepsilon)}{\log \varepsilon / \varepsilon_{0}}+$ const,

where $\varepsilon_{0}$ is the arbitrary length.

Furthermore, the RQA can be used to identify topological structures of diagonal and vertical lines. In its frame, RQA provides us with the probability $p(l)$ or $p(v)$ of line distribution according to their lengths $l$ or $v$ (for diagonal and vertical lines). Practically they are calculated as

$p(x)=\frac{P^{\varepsilon}(x)}{\sum_{x=x_{\min }}^{N} P^{\varepsilon}(x)}$,

where $x=l$ or $v$, depending on diagonal or vertical structures in the specific recurrence diagram. $P^{\varepsilon}(x)$ denotes the unnormalized probability for a given threshold value $\varepsilon$. In this way, the Shannon information entropies $\left(L_{\mathrm{ENTR}}\right)$ can be defined for diagonal line collections:

$L_{\mathrm{ENTR}}=-\sum_{l=l_{\min }}^{N} p(l) \ln p(l)$.

Other properties, the determinism DET and laminarity $L A M$, are defined as
Fig. 9 Recurrence plots for regular (a) and irregular (b) solutions for the same system parameters as point " 1 " and " 2 " in Figs. 4a and $4 \mathrm{~b}$, respectively $(\varepsilon=0.01)$
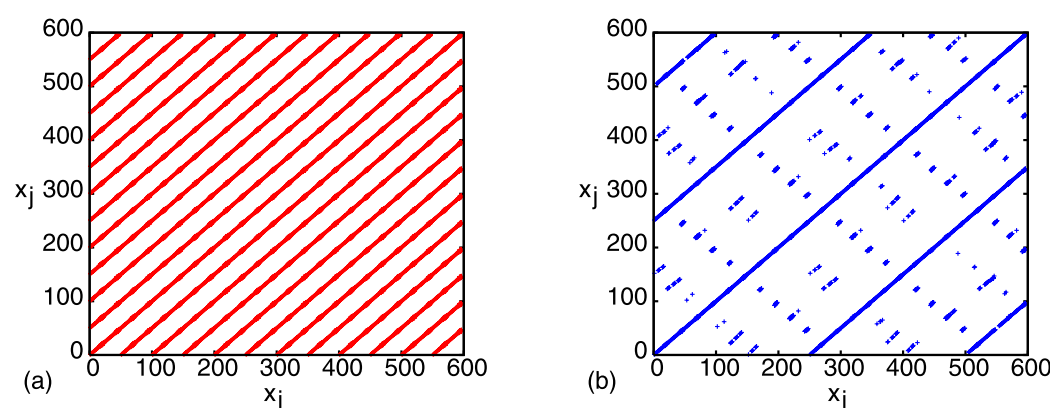
Table 1 Summary of recurrence quantification analysis (RQA) for $m=2$ and $\varepsilon=0.01$ for " 1 " and "2" solutions (see Figs. 4, 5 and 6)

\begin{tabular}{lllll}
\hline Type of motion & $R R$ & $D E T$ & $L A M$ & $L_{\text {ENTR }}$ \\
\hline (“1”) & 0.0348 & 1.0000 & 0.6078 & 1.3135 \\
(“2”) & 0.0080 & 0.9578 & 0.4743 & 1.7928 \\
\hline
\end{tabular}

$D E T=\frac{\sum_{l=l_{\min }}^{N} l P^{\varepsilon}(l)}{\sum_{i, j=1}^{N} l P^{\varepsilon}(l)}$,

$L A M=\frac{\sum_{v=v_{\min }}^{N} v P^{\varepsilon}(v)}{\sum_{v=1}^{N} v P^{\varepsilon}(v)}$,

where $l_{\min }$ and $v_{\min }$ denote the minimal values which should be chosen for a specific dynamical system. In our calculations we have assumed that $l_{\min }=$ $v_{\min }=2$.

The determinism DET is a measure of the predictability of the examined time series and gives the ratio of recurrent points formed in diagonals to all recurrent points. Note that in a periodic system all points should be included in the lines. On the other hand, the laminarity $L A M$ is a similar measure which corresponds to points formed in vertical lines. This measure indicates the dynamics behind sampling point changes.

The results of our analysis calculated for time series "1" and "2" (see Figs. 4, 5 and 6) are presented in Fig. 9, where we present the results of RP for $\varepsilon=0.01$. Note that both plots show regular features. However, Fig. 9a is composed of full diagonals lines only, while in Fig. 9b each fifth line is full, and between them one observes short line pieces or even insulated points. This would indicate a multi-frequency modulated solution. There can be also a transient with basic regular and superimposed chaotic solutions. To shine this difference with more lights, we show some estimated RQA parameters in Table 1. Obviously, $R R$ is smaller for "2" (Fig. 9b) as we have broken lines instead of full ones (Fig. 9a). Moreover, the determinism and laminarity (DET and $L A M)$ are smaller, telling us that the system is less regular. Consequently the more peculiar distribution of line lengths is confirmed by $L_{\text {ENTR }}$, which is larger for the solution " 2 ". Additionally, in Fig. 10 we present the results of $R R$ versus $\varepsilon$ (for relatively small $\varepsilon$ ). One can see a significant difference between both solutions.

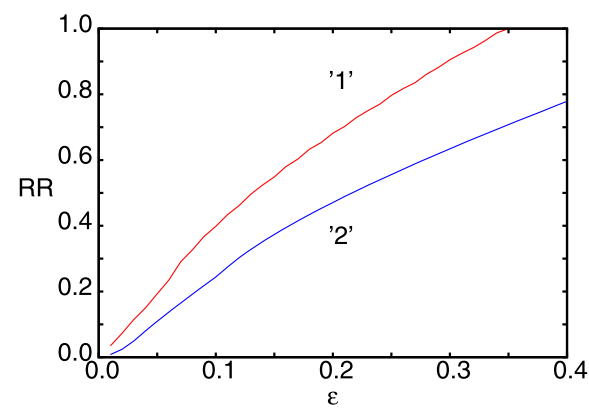

Fig. 10 Recurrence rate $R R$ versus threshold value $\varepsilon$ for regular " 1 " and irregular " 2 " solutions (" 1 " and " 2 " as in Figs. 4a and $4 \mathrm{~b}$, respectively)

\section{Summary and conclusions}

We have analyzed the two-DOF quarter-car model, assuming that damping and suspension through the unsprung mass excited by the road profile corrugation can act as a perturbation on the main sprung mass. The obtained Melnikov criterion was latter confirmed by numerical simulations. The main conclusion coming from that point would be loss of stability of the system appearing as the chaotic or transient chaotic or escape solution. The present investigation is going beyond research dealing with a single-DOF quarter-car model $[4,18,19,24]$. In particular, the single-DOF model assumes that the unsprung mass is significantly smaller than the sprung mass.

One should note that the model used in this paper, although more realistic than the previous singledegree-of-freedom ones, is relatively simple and would not be sufficient to simulate a detailed response of a vehicle or compare to experimental results from real vehicles. Unfortunately, more sophisticated half-car and full-car models [31-33] cannot be used in the frame of the presented approach as the heteroclinc trajectories could not be defined reliably. Furthermore, in higher-DOF systems the analytic perturbation calculations are not possible.

However, the present quarter-car model is able to capture the major nonlinear effects that occur in vehicle dynamics and has demonstrated the transition to chaotic vibrations and synchronization phenomena $[25,32,39]$. Interestingly, the resulting critical amplitude curve (Fig. 4a, Eq. (19)) has the maximum for $\omega \approx 3.1$ and the minimum for $\omega \approx 2.2$. The minimum is obviously related to the resonance region of the decoupled unsprung solution $x_{2}$ (Eqs. (8) and (9)). 
It should be also noted that the recurrence plot technique appeared to be very useful to study the transient signals. Thus, the conclusions that came from the analytic approach have been confirmed. Indeed, this method is designed for the short time series $[44,49]$ Interestingly, it also works for nonstationary signals $[44,48]$. The recurrences for RP and RQ analyses have been obtain using the available command line code written by Marwan [50]. The recurrence approach can be also used to higher-DOF models of vehicle dynamics. The corresponding results on half- and full-vehicle models will reported in a separate paper.

Acknowledgements Authors thank Prof. Lenci for fruitful discussions. The research leading to these results has received funding from the European Union Seventh Framework Programme (FP7/2007-2013), FP7-REGPOT-2009-1, under grant agreement No: 245479.

Open Access This article is distributed under the terms of the Creative Commons Attribution License which permits any use, distribution, and reproduction in any medium, provided the original author(s) and the source are credited.

\section{References}

1. Verros, G., Natsiavias, S., Stepan, G.: Control and dynamics of quarter-car models with dual-rate damping. J. Vib. Control 6, 1045-1063 (2000)

2. Gobbi, M., Mastinu, G.: Analytical description and optimization of the dynamic behaviour of passively suspended road vehicles. J. Sound Vib. 245, 457-481 (2001)

3. Von Wagner, U.: On non-linear stochastic dynamics of quarter car models. Int. J. Non-Linear Mech. 39, 753-765 (2004)

4. Li, S., Yang, S., Guo, W.: Investigation on chaotic motion in hysteretic non-linear suspension system with multifrequency excitations. Mech. Res. Commun. 31, 229-236 (2004)

5. Litak, G., Borowiec, M., Kasperek, R.: Response of a magneto-rheological fluid damper subjected to periodic forcing in a high frequency limit. Z. Angew. Math. Mech. 88, 1000-1004 (2008)

6. Naik, R.D., Singru, P.M.: Establishing the limiting conditions of operation of magneto-rheological fluid dampers in vehicle suspension systems. Mech. Res. Commun. 8, 957 962 (2009)

7. Turkay, S., Akcay, H.: A study of random vibration characteristics of the quarter-car model. J. Sound Vib. 282, 111124 (2005)

8. Verros, G., Natsiavas, S., Papadimitriou, C.: Design optimization of quarter-car models with passive and semiactive suspensions under random road excitation. J. Vib. Control 11, 581-606 (2005)

9. Li, S., Yang, S.: Chaos in vehicle suspension system with hysteretic nonlinearity. J. Vib. Meas. Diagn. 23, 86-89 (2003)
10. Shen, Y., Yang, S., Chen, E., Xing, H.: Dynamic analysis of a nonlinear system under semi-active control. J. Vib. Eng. 18, 219-222 (2005)

11. Yang, S., Li, S., Guo, W.: Chaotic motion in hysteretic nonlinear suspension system to random excitation. J. Vib. Meas. Diagn. 25, 22-25 (2005)

12. Yang, S., Li, S., Wang, X., Gordaninejad, F., Hitchcock, G.: A hysteresis model for magneto-rheological damper. Int. J. Nonlinear Sci. Numer. Simul. 6, 139-144 (2005)

13. Pan, C., Yang, S., Shen, Y.: An electro-mechanical coupling model of magnetorheological damper. Int. J. Nonlinear Sci. Numer. Simul. 6, 69-74 (2005)

14. Genta, G.: Motor Vehicle Dynamics. World Scientific, Singapore (2003)

15. Andrzejewski, R., Awrejcewicz, J.: Nonlinear Dynamics of a Wheeled Vehicle. Springer, New York (2005)

16. Gao, G., Yang, S., Chen, E., Xing, H.: A study on the modeling of magnetorheological dampers for vehicle suspension based on experiment. Automob. Eng. 26, 683-685 (2004)

17. Gao, G., Yang, S., Chen, E., Guo, J.: One local bifurcation of nonlinear system based on magnetorheological dampers. Acta Mech. Sin. 36, 564-568 (2004)

18. Yang, S., Li, S.: Primary resonance reduction of a singledegree-of-freedom system using magnetorheological fluid dampers. J. Dyn. Control 2, 62-66 (2004)

19. Litak, G., Borowiec, M., Friswell, M.I., Szabelski, K.: Chaotic vibration of a quarter-car model excited by the road surface profile. Commun. Nonlinear Sci. Numer. Simul. 13, 1373-1383 (2008)

20. Siewe, M.S.: Resonance, stability and period-doubling bifurcation of a quarter-car model excited by the road surface profile. Phys. Lett. A 374, 1469-1476 (2010)

21. Naik, R.D., Singru, P.M.: Resonance, stability and chaotic vibration of a quarter-car vehicle model with time-delay feedback. Commun. Nonlinear Sci. Numer. Simul. 16, 3397-3410 (2011)

22. Wu, C., Wang, W.-R., Xu, B.-H., Li, X.-L., Jiang, W.-G.: Chaotic behavior of hysteretic suspension model excited by road surface profile. J. Zhejiang Univ. Eng. Sci. 45, 12591264 (2011)

23. Li, C., Liang, S., Zhu, Q., Xiong, Q.: Analysis of chaotic vibration of a nonlinear quarter-vehicle model caused by the consecutive speed control humps. ICIC Express Lett. 5, 3201-3207 (2011)

24. Litak, G., Borowiec, M., Friswell, M.I., Przystupa, W.: Chaotic response of a quarter-car model excited by the road surface profile with a stochastic component. Chaos Solitons Fractals 39, 2448-2456 (2009)

25. Borowiec, M., Hunicz, J., Sen, A.K., Litak, G., Koszalka, G., Niewczas, A.: Vibrations of a vehicle excited by real road profiles. Forsch. Ingenieurwes. 74, 99-109 (2010)

26. Ciocogna, G., Popoff, F.: Asymmetric duffing equation and the appearance of chaos. Europhys. Lett. 3, 963-967 (1987)

27. Szemplinska-Stupnicka, W.W.: The refined approximate criterion for chaos in a two-state mechanical systems. Ing.Arch. 58, 554-566 (1988)

28. Brunsden, V., Coetell, J., Holmes, P.: Power spectra of chaotic vibrations of a buckled beam. J. Sound Vib. 130, 561-577 (1989) 
29. Thompson, J.M.T.: Chaotic phenomena triggering the escape from a potential well. Proc. R. Soc. Lond. Ser. A, Math. Phys. Sci. 421, 195-225 (1989)

30. Bruhn, B., Koch, B.P., Schmidt, G.: On the onset of chaotic dynamics in asymmetric oscillators. Z. Angew. Math. Mech. 74, 325-331 (1994)

31. Zhu, Q., Ishitobi, M.: Chaos and bifurcations in a nonlinear vehicle model. J. Sound Vib. 275, 1136-1146 (2004)

32. Zhu, Q., Ishitobi, M.: Chaotic vibration of a nonlinear fullvehicle model. Int. J. Solids Struct. 43, 747-759 (2006)

33. Wang, W., Li, G., Song, Y.: Nonlinear dynamic analysis of the whole vehicle on bumpy road. Trans. Tianjin Univ. 16, 50-55 (2010)

34. Melnikov, V.K.: On the stability of the center for time periodic perturbations. Trans. Mosc. Math. Soc. 12, 1-57 (1963)

35. Guckenheimer, J., Holmes, P.: Nonlinear Oscillations, Dynamical Systems and Bifurcations of Vector Fields. Springer, New York (1983)

36. Wiggins, S.: Introduction to Applied Nonlinear Dynamical Systems and Chaos. Springer, New York (1990)

37. Seoane, J.M., Zambrano, S., Marino, I.P., Sanjuan, M.A.F. Basin boundary metamorphoses and phase control. Europhys. Lett. 90, 30002 (2010)

38. Kwuimy, C.A.K., Nataraj, C., Litak, G.: Melnikov's criteria, parametric control of chaos, and stationary chaos occurrence in systems with asymmetric potential subjected to multiscale type excitation. Chaos 21, 043113 (2011)

39. Borowiec, M., Litak, G., Friswell, M.I.: Nonlinear response of an oscillator with a magneto-rheological damper subjected to external forcing. Appl. Mech. Mater. 5-6, 277$284(2006)$
40. Ruzziconi, L., Litak, G., Lenci, S.: Nonlinear oscillations, transition to chaos and escape in the Duffing system with non-classical damping. J. Vibroeng. 13, 22-38 (2011)

41. Eckmann, J.-P., Kamphorst, S.O., Ruelle, D.: Recurrence plots of dynamical systems. Europhys. Lett. 5, 973-977 (1987)

42. Casdagli, M.C.: Recurrence plots revisited. Physica D 108, 12-44 (1997)

43. Marwan, N.: Encounters with neighbours: current development of concepts based on recurrence plots and their applications. PhD Thesis, Universität Potsdam, Potsdam (2003)

44. Marwan, N., Romano, M.C., Thiel, M., Kurths, J.: Recurrence plots for the analysis of complex systems. Phys. Rep. 438, 237-329 (2007)

45. Webber, C.L. Jr., Zbilut, J.P.: Dynamical assessment of physiological systems and states using recurrence plot strategies. J. Appl. Physiol. 76, 965-973 (1994)

46. Marwan, N.: A historical review of recurrence plots. Eur. Phys. J. Spec. Top. 164, 3-12 (2008)

47. Nichols, J.M., Trickey, S.T., Seaver, M.: Damage detection using multivariate recurrence quantification analysis. Mech. Syst. Signal Process. 20, 421-437 (2006)

48. Litak, G., Longwic, R.: Analysis of repeatability of diesel engine acceleration. Appl. Therm. Eng. 29, 3574-3578 (2009)

49. Litak, G., Wiercigroch, M., Horton, B.W., Xu, X.: Transient chaotic behaviour versus periodic motion of a pendulum by recurrence plots. Z. Angew. Math. Mech. 90, 33-41 (2010)

50. Marwan, N.: Commandline recurrence plots (2011). http:// www.agnld.uni-potsdam.de/marwan/6.download/rp.php (visited 2011-08-15) 